\title{
Learning Analytics in Flipped Classrooms: A Scoping Review
}

\author{
Muriel Algayres and Evangelia Triantafyllou \\ Aalborg University, Copenhagen, Denmark \\ mgal@create.aau.dk \\ evt@create.aau.dk \\ DOI: 10.34190/JEL.18.5.003
}

\begin{abstract}
The Flipped Classroom (FC) is an instruction method, where the traditional lecture and homework sessions are inverted. Online material is given to students in order to gain necessary knowledge before class, while class time is devoted to application of this knowledge and reflection. The hypothesis is that there could be deep and creative discussions when teacher and students physically meet, which has known a significant surge of popularity in the past decade. A marked recent trend in the FC is the increased use of Learning Analytics (LA) to support the development of the FC and students' reflexive learning. The aim of this paper is to investigate the literature on applications of LA in FCs, and to determine the best practices and needs for technological development supporting LA in the FC by means of a scoping review. This literature review revealed that there is potential in using LA in the FC, especially as a means to predict students' learning outcome and to support adaptive learning and improvement on the curriculum. However, further long-term studies and development is necessary to encourage self-directed learning in students and to develop the whole of the FC for a more diverse population of students. We anticipate an increased and expanded use of LA to come, with focus on predictive and prescriptive analytics providing more adaptive learning experience. We also anticipate that LA will expand beyond data mining to correlate student performance and online engagement with the aim to include a wider range of possibilities of interventions and adaptation of the learning experience.
\end{abstract}

Keywords: active learning, flipped classroom, learning analytics, virtual learning environment, educational data mining

\section{Introduction}

The Flipped Classroom (FC) is possibly one of the most prominent endeavors to overhaul educational practices in recent years. Faced with the need to engage students, and with disaffection from the traditional lecture-based model, educational institutions turned towards active learning to shift "...the focus of learning from passively receiving content information to diligently participating in learning activities" (Frey, 2018). In that regard, the FC, where "...events that have traditionally taken place inside the classroom now take place outside the classroom and vice versa" (Lage, Platt and Treglia, 2000) is a concrete application of the principles of active learning. After a first decade of developments and tests of the model, the FC is undergoing further developments, amongst which the increased use of Learning Analytics (LA) (Fernández, Merino and Kloos, 2018).

\subsection{The Flipped Classroom}

Interest for the FC however rose sharply in the early 2010s, following its popularization in secondary education in the Unites States (Bergmann and Sams, 2009). It is now frequent in higher education, as a means to engage an increasingly diverse and flexible population of students (Reidsema et al., 2017). Abeysekera and Dawson (2015) provided a "lowest common denominator" definition, defining the FC as "a set of pedagogical approaches that: (1) move most information-transmission teaching out of class, (2) use class time for learning activities that are active and social, and (3) require students to complete pre- and/or post-class activities to fully benefit from in-class work."

The FC methodology has been frequently reviewed and studied, and interest in the methodology has remained constant in the past decade (Bishop and Verleger, 2013; O'Flaherty and Phillips, 2015; Zainuddin and Halili, 2016). The FC has been recognized as an effective learning approach in various courses: it gives teachers more time for personalized interactions with students, improves students' creative thinking and communication skills, and encourages students' responsibility in their learning (Lin and Hwang, 2018).

\subsection{Learning analytics}

Based on the most commonly cited definition, "LA is the measurement, collection, analysis and reporting of data about learners and their contexts, for purposes of understanding and optimizing learning and the environments in which it occurs." (Siemens and Long, 2011). Figure 1 presents the different steps in the LA process. 


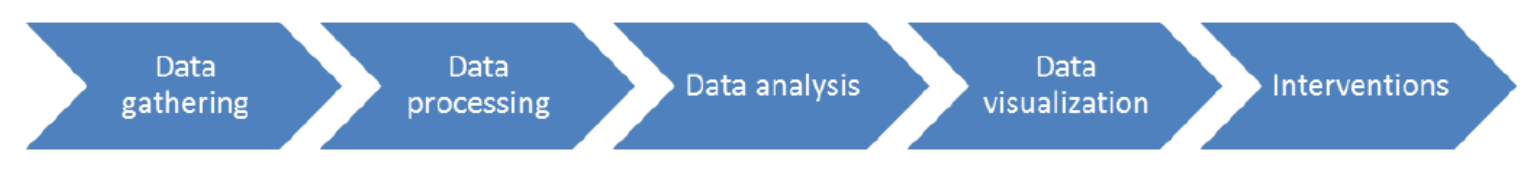

Figure 1: The different steps in the LA process

LA aim at providing ways to gather and make sense of educational data, which is generated while educators and learners interact with digital technologies. The goal is to improve the learning experience for learners and teachers, and better adapt courses' design. Although LA is a relatively young field, it might prove crucial in further developments of the FC since it can inform teachers about the learning process of the students, and teachers can in turn use this information to make informed pedagogical decisions (Van Leeuwen, 2018). The method also encourages adaptive learning and self-regulated learning: learners can improve their meta-cognitive abilities with information to reflect on their own learning, and teachers can create a feedback loop between online and classroom phases to assess the progress and needs of students (Klemke, Eradze and Antonaci, 2018).

In a recent study of LA trends based on more than a hundred articles, Leitner, Khalil, and Ebner (2017) anticipated that LA would continue to be a staple of higher education. They argued that in the short term the potential to cater to individual learning outcomes, and personalized feedback and visualization would increase, and that the rise of predictive analysis would mark long-term trends. Although this evolution appears slower than originally stated, these issues remain central to the development of LA.

\subsection{Combining the flipped classroom and learning analytics}

Although both the FC and LA have both been active fields of study, research in combining them is still rare. According to Fernández, Merino and Kloos (2018), early research focused on the results obtained in the FC, or used specific indicators to obtain useful information for the FC. However, that research did not indicate precisely what the best tools were, or what specific learning activities they could support.

More recently, the potential of using LA in the FC has come to increased focus and attention. For example, Doko and Bexheti (2018) tried to map educational data mining practices with a wide scope, including MOOCs, videos, and the FC. Their study showed an increased number of studies after 2010 in these issues. While their study provided a comprehensive overview of interest in educational data mining, it was constrained by a very wide scope that ended up diluting the specific issue of LA in the FC in an approach where Video-Based Learning (VBL) dominate. As such, this study showed interest for LA in the FC but did not allow a targeted understanding of the processes at work, when we attempt to analyze the use of LA in the FC. Similarly, Lam, Lau, and Chan (2019) recently advocated extended use of LA in the FC while pointing out the limitations of current Learning Management Systems (LMSs) to provide a valuable use of LA in the FC. They argued that LA has the potential both to assess the student's learning process, provide feedback for the student's improvement, and offer data for the teacher's reflection, but that current assessment practices and reliance on LMSs limit the full potential of LA.

This article means therefore to complete the existing literature by examining the potential in combining the FC and LA through a scoping review of the existing literature. We believe that examining previous use of LA in the FC will allow us to determine when LA have been used successfully, and where use of LA is still lacking. There are several advantages to using a scoping review. According to Arksey and O'Malley (2005), it allows researchers to examine the extent, range, and nature of the research activity, to determine the value for undertaking a full systematic review, to summarize and disseminate research findings, and to identify gaps in existing literature. We will therefore research the implementation of LA in the FC, and determine the best practices, the limitations, and ways to improve it.

\section{Methodology}

For this paper, we have used Arksey and O'Malley's methodology (2005), as expanded by Levac et al. (2010). The scoping review method uses the stages presented in the following sections. 


\subsection{Stage 1: Identifying the research question}

The focus of this research is to explore the key factors in the use LA in the FC. We also want to examine how LA can improve the FC model. To ensure that a substantial range of literature was examined, we followed the following research questions to guide the search:

1. Which type of data and learning analytics algorithms were used in the FC and to what purpose?

2. What were the educational outcomes of using LA in the FC, and which theoretical background were they based upon?

3. What were the main limitations in the use of LA in the FC?

\subsection{Stage 2: Identifying relevant studies}

Arksey and O'Malley suggest that a wide definition for search terms should be used. Therefore, we opted for a large selection of related terms, which covered various forms of LA. The following research string was thus devised:

("flipped classroom") AND (("learning analytics") OR ( "engagement data") OR ("educational data" ) OR ("activity data" ) OR ("data mining")). The selection was then restricted to research that specifically used LA in the FC.

The selected databases for this study were Scopus, Proquest, Web of Science, and JSTOR. Only peer-reviewed articles and papers, accessible in English, were selected. The research was carried for the period 2009-2019, a decade, which coincides with the exponential development of the FC.

\subsection{Stage 3: Study selection}

Using the key search descriptors, we identified an initial selection of 188 articles. Figure 2 presents the selection process and exclusion criteria to the selection.

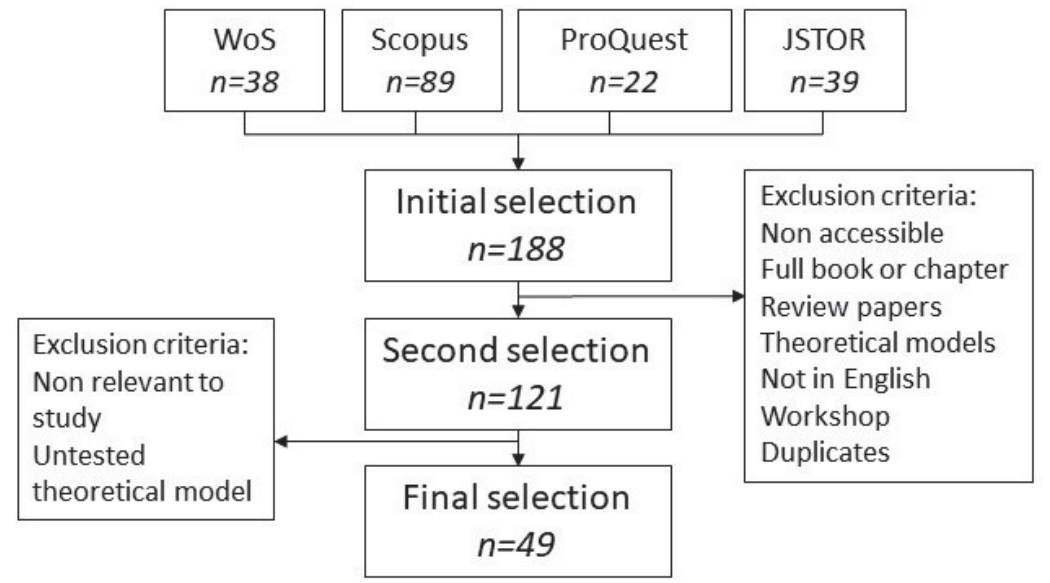

Figure 2: Model for the article selection

The first round of exclusion took out duplicates and formats that did not fit the research, narrowing the selection to 121 articles. From these, a detailed reading of abstracts allowed us to select studies who were actually combining LA as a tool in the FC, and not addressing both methodologies as separate subjects, or in untested theoretical models. From there, we finally ended with a final selection of 49 articles.

\subsection{Stage 4: Charting the data}

The data extracted from the selection of articles was mapped using the following attributes: Study ID, Database, Paper title, Journal/proceeding, Author, Year, Country of study, LA algorithms, Data extracted, Feedback to students, Position in the FC, Level of class, Subject, Size of class, Control group (if applies), Outcomes, Evaluated variables, Methodology, Evaluation of performance (grade, knowledge test, learning outcomes), Evaluation of students perception (self-reported opinion, interviews, interest, attitudes), Student experience (motivation, stress level, engagement, participation, cognitive load), Theoretical framework, Limitations. 


\subsection{Stage 5: Collating, summarizing and reporting the results}

The final stage of the scoping review summarizes and reports findings.

\section{Findings}

The publishing information for the studies we selected yielded several observations. A first analysis of the selected studies showed that the issue of LA was a recent subject, who met an increased interest in the second part of the observed decade. Figure 3 represents the distribution of studies by year.

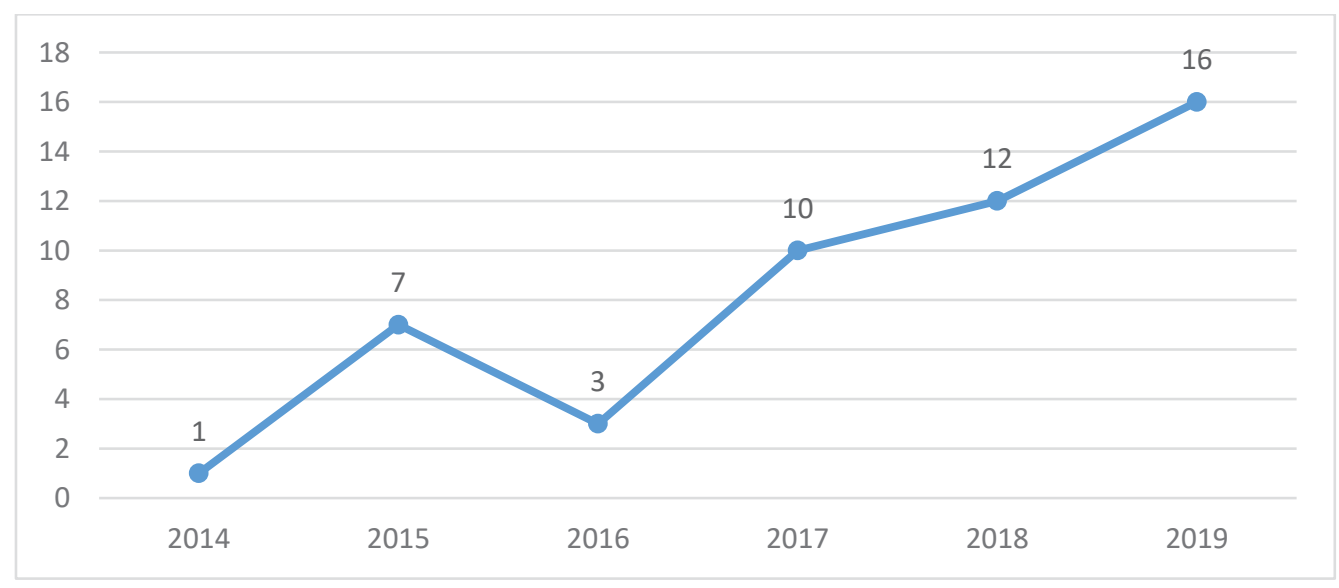

Figure 3: Distribution of studies by year of publication

We can observe that studies on LA have increased in the past three years, with a consolidation of publications after 2017. The geographic distribution of studies, however, appeared more homogenous: 18 countries were represented, nine in Europe, two in South America, four in Asia, as well as the USA and Australia. Only five countries had more than five articles: the USA (nine articles), China including Hong Kong (seven articles), Taiwan (six articles), Australia and Spain (five articles each). The rest presented only one or two studies.

The type of study presented a stark domination of STEM in higher education. Only four studies interested themselves to secondary education, and the other 45 studied classes at university level. Figure 4 presents the distribution of articles by subject. We can observe the domination of STEM related subjects: Computer science (11 articles), Engineering (11 articles), Math (6 articles) and other scientific subjects e.g. physics or biology (7 articles). This data explains these discrepancies: as learning systems that integrate LA are a recent development, early testing was carried in the departments that already had the technical competencies in learning algorithms and machine learning. It will be noted, however, that a greater scope of investigation remains to be explored to adapt LA to wider contexts.

Finally, only one study (Hsu, 2019) specifically investigated LA during the in-class activity time. The rest of the articles were evenly distributed between pre-class preparation (27 articles), and the whole FC with a focus on out-of-class student monitoring (21 articles). 


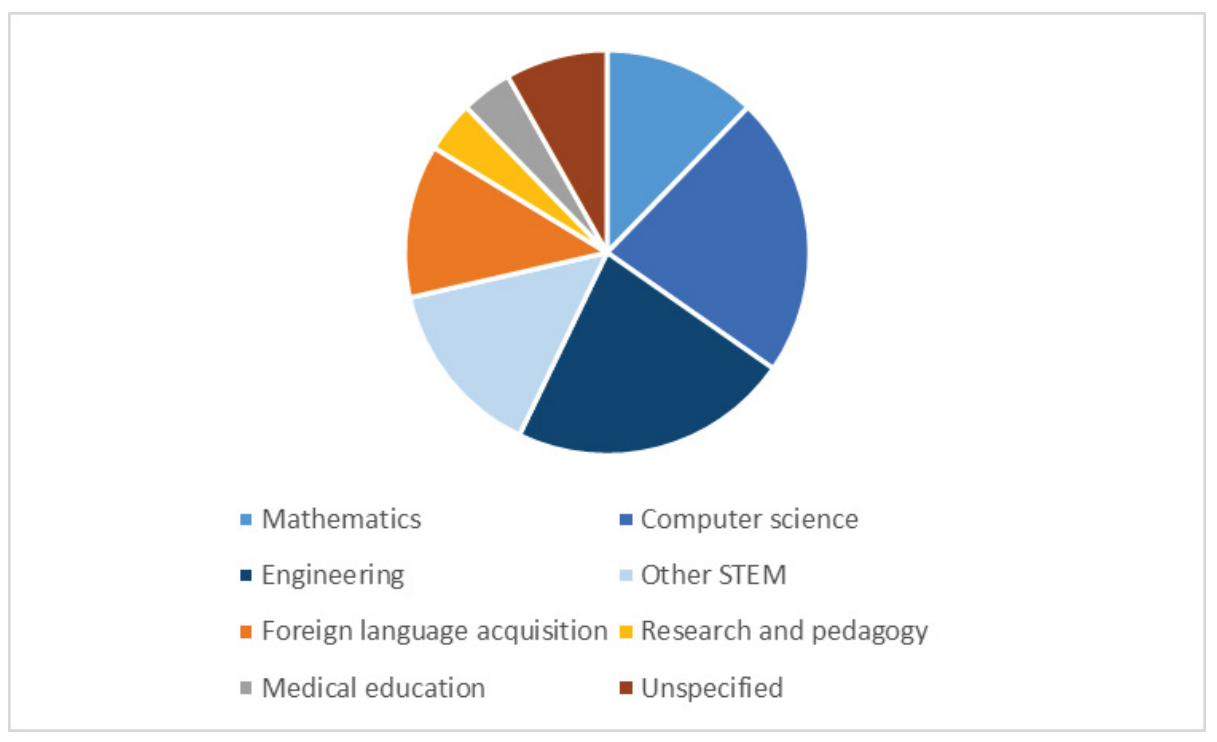

Figure 4: Distribution of studies by subject

\subsection{Which type of data and learning algorithms can be used to implement LA in the FC?}

To further this analysis, we also observed which model of data extraction, and which learning algorithms were used in the selected studies. Every study tended to use more than a single approach in extracting relevant data, as well as applying different algorithms to the data set. Therefore, the numbers in this section will exceed our initial 49 , but will reflect the common denominators in the choices that have prevailed thus far in applying LA. Figure 5 presents the modalities of data extraction.

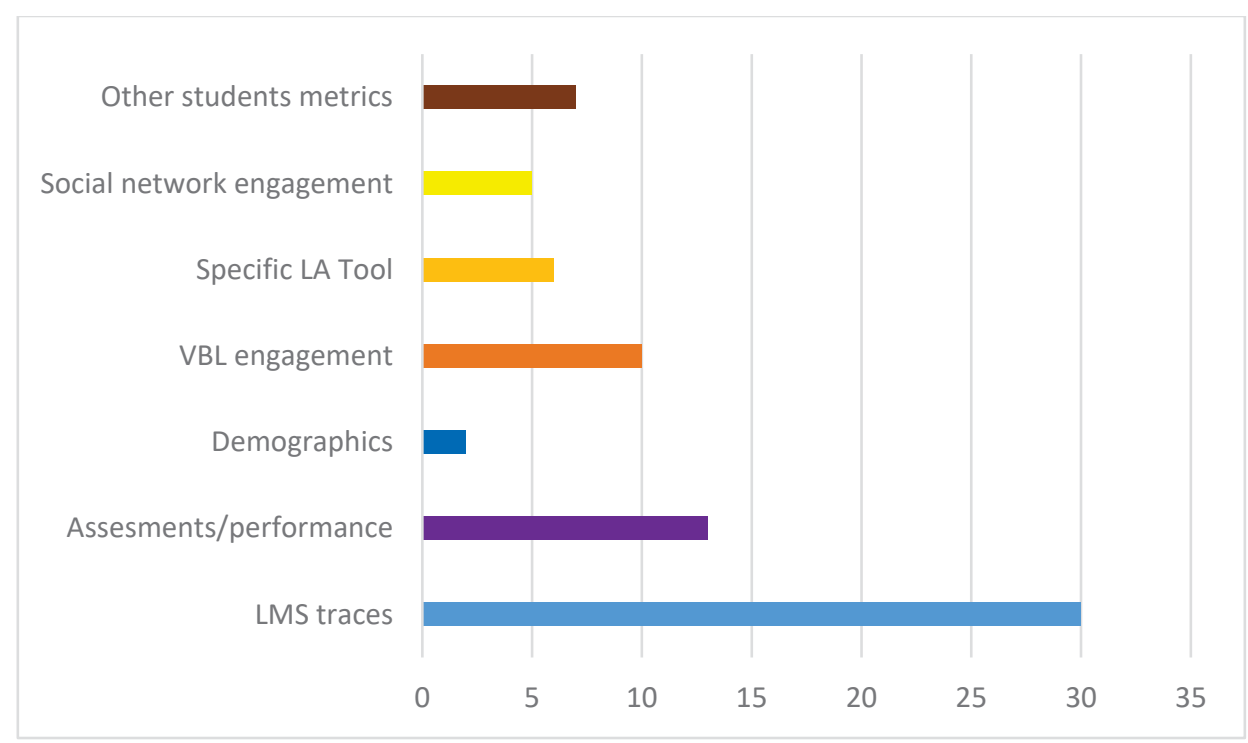

Figure 5: Type of data extraction for LA in the FC studies (by number of studies)

Data mining and exploitation of LMS traces, especially in Moodle, is the main source of data in the articles we reviewed, appearing in 30 articles (e.g. Gelan et al., 2018; Yamada and Hirakawa, 2015; Lin, 2019). LMS traces focus usually on student engagement with the learning platform, and usually cover such basics as time spent on lesson (Matcha et al., 2019; Martínez et al., 2019), completion time of online activities (e.g. Poon et al., 2017; Ayres et al., 2018), and regularity and frequency of engagement with the platform (e.g. Jovanovic et al., 2019).

Stemming from the previous category, we also identified 13 studies that specifically focus on online performance leading to an evaluation or assessment. Metrics such as the completion rate and success rate in the course are taken into account (Kaw, et al. 2019; Yang, Wu and Cao, 2016), as well as summative evaluations (Smallhorn, 2017). A single study integrated peer evaluation (Fernández, Merino and Kloos, 2019). 
A significant number of studies focused on the pre-class preparation through engagement with the video lectures, with 10 articles (e.g. Xiao, Pham and Wang, 2015). In these articles, the focus was on data as interactive notetaking (Hecking et al., 2017; Pardo et al., 2015), results from embedded multiple choices questionnaires in the video (e.g. Giannakos, Chorianopoulos and Chrisochoides, 2015), multiple views and unique viewers per video (Gilliland, 2017), or assessment of the video quality by the learners (Giannakos and Chrisochoides, 2014).

Five studies focused on social interactions as a means to engage learners in the learning process, using data such as their comments on the class Facebook page (Lin and Hwang, 2018), the online interactions with peers or teachers (Ji and Han, 2019; Reidsema et al., 2017), or the volume of submissions on the learning system (Isomöttönen and Tirronen, 2017). Another study used specific applications on the students' mobile phones to evaluate work on a BYOD (Bring Your Own Device) configuration, where students were encouraged to use social networks for support (Hsu, 2019).

Some articles focused on other student metrics, such as student self-reported satisfaction in the experience (Lei et al., 2017; Van Leeuwen, 2018), student self-assessment (Corrias and Hong, 2015), or self-regulation in learning (Hwang and Chen, 2019). Two studies investigated the students' adaptive learning pathway in the FC experience (Kaw and al., 2019; Reidsema et al., 2017). One study (Xiao, Pham and Wang, 2015) introduced a rare example of a mobile MOOC, where learning was adapted via implicit cognitive states inference that integrated a tangible video control, and implicit heart rate sensing based on heartbeat waveforms.

Finally, a minority of six articles presented specific data mining, and LA tools that were devised to facilitate that process. For example, Mouri, Uosaki and Ogata (2018) presented an e-book using a ubiquitous learning system called SCROLL, and a specific LA tool, VASCORLL 2.0 (Visualization and Analysis System for COnnecting Relationships of Learning Logs). Redondo et al. (2015) used the plug-in tool ANALYSE to handle their LA. Similarly, Xiao et al. (2015) used their own custom system, AttentiveLearner. Fernández, Merino, and Kloos (2019) used a custom learning platform and LA tool (called GE-L+), and Martínez et al. (2019) the learning engagement tool Socrative. Other examples include specific tools used on students' mobile phones (Hsu, 2019), and an unnamed CLI (Command Line Interface) data extraction tool (Ayres et al., 2018).

While a small majority of studies used only quantitative data as mentioned above, 21 studies used mixed methods and completed their use of LA with qualitative data. Such studies investigated the participants' experience through interviews (e.g. Hui et al., 2018; Kravchenko and Cass, 2017) or focus groups (e.g, Ayres et al, 2018; Kaw et al., 2019), or in-class observation based on the action research methodology (Isomöttönen and Tirronen, 2017). Some studies also investigated the lived experiences of students through self-reported opinion or motivation questionnaires (Marasco et al., 2018; Lau et al., 2018; Lei et al., 2017; Chu, Wang and Wang, 2019). Sun, Lu and Xie (2019) introduced results from Motivated Strategies for Learning Questionnaires (MSLQ), and Hwang and Chen (2019) analysed the content in questionnaires with a Quantitative Content Analysis (QCA) to evaluate the students' experience.

However, although mixed methods are applicable to the observation of the FC experience, the core of the data was quantitative and used to apply LA algorithms. Figure 6 presents the type of algorithm applied in the LA phase. Similarly, most articles used more than one type of algorithm to conduct their study so the results go beyond our original 49 occurrences. We have sorted the use of LA in the following categories: cluster analysis, sequential analysis, predictive analysis, linear regression analysis, data mining, process mining, and other nonclassified in the previous categories approaches. This classification relies on the terminology of the studies themselves, while unspecified mentions of LA fell under data mining. The order of importance in which these diverse approaches occurred is the following:

- Data mining refers to projection based on participants' engagement in the learning platform, essentially based on LMS traces. These cover essentially log-ins to the platform, number of resources accessed, time spent on resources, number of clicks, number of exercises taken, and completion rates.

- Cluster analysis appears to determine profile groups of students based on their achievement rates or level of engagement with the platform. These diagnostics allow determining learner profiles or group dynamics. For example, Sun, Lu, and Xie (2016) managed to identify up to six learning groups using cluster analysis.

- $\quad$ Predictive statistics were used to correlate student profiles with student results or pass grade, and try to predict students' success or dropout risk based on their learning profile, and engagement in the FC and online activity (Hui and al., 2018) 
- Sequential analysis means to analyse patterns of engagement based in time. It focuses on the distribution and frequency of online activities, and its articulation with in-class time and assessments. The minor category process mining employs the same data to determine learning strategies in conjunction with cluster analysis. For example, Fincham et al. (2018) used a combination of cluster analysis and sequential analysis to determine which learning patterns were more efficient for students.

- Linear regression analysis is used in these studies to establish relations between variables in the data mining process, usually between student level of engagement and their pass rates, or in the case of mixed method studies, with the students' comments and experiences. For example, Hsiao et al. (2018) used multiple linear regression to show that students' online learning behaviour did not have a shortterm impact on learning outcome, but did have a significant long-term impact.

- The unclassified category covers models that only appear in two studies or less. However, we can underline that these minority studies offer often more complex statistical models in the form of neural network and Bayesian models, trying to ascertain complex patterns in the learner behaviour (e.g. Nouri, Saqr, and Fors, 2019).

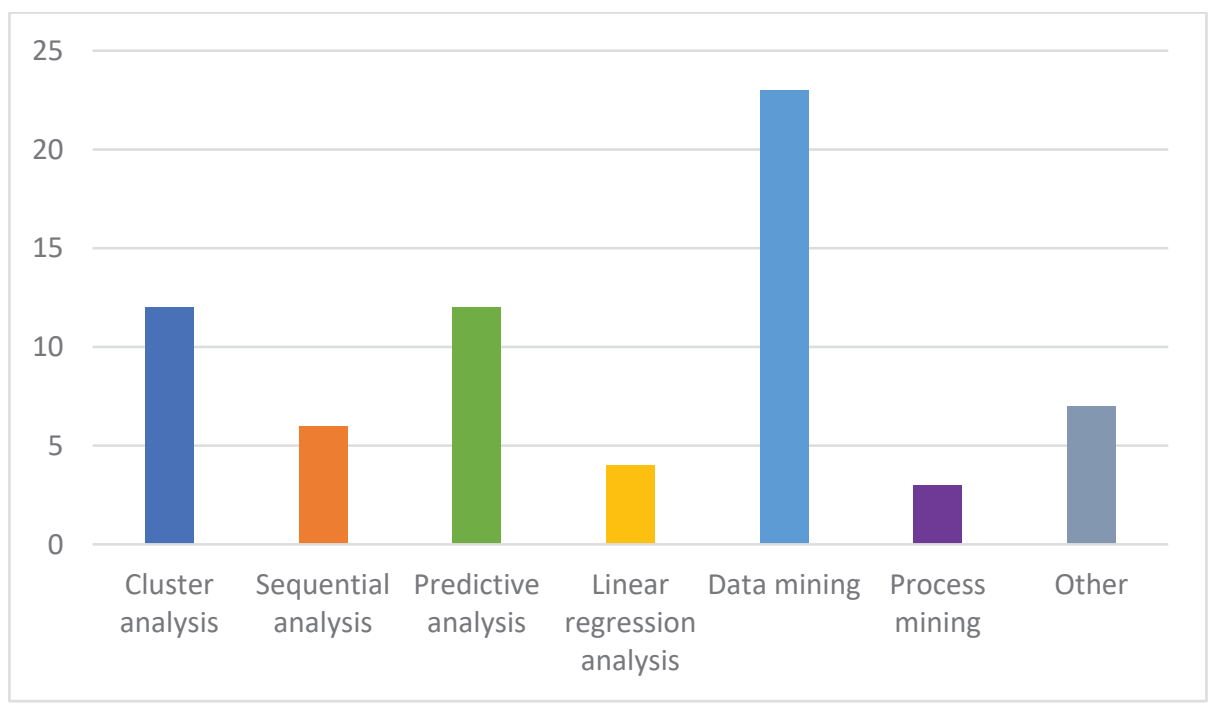

Figure 6: Type of LA (by mention in number of studies)

Therefore, there is a very clear domination in the studies of LMS and VBL traces used for descriptive or diagnostic analytics. However, LA is a quickly expanding field and other forms of cluster and predictive analysis are clearly catching up, while use of original and dedicated LA tools start to encroach in recent studies.

\subsection{What were the educational outcomes of using LA in the FC, and which theoretical background were they based upon?}

We then looked into what was the basis on which these studies established their conclusion. Where all studies presented LA and the FC, owing to the terms of our initial research, only a smaller part of our study corpus expanded their theoretical backgrounds beyond framing the context. Figure 7 presents a tentative description of the theoretical frame for integration of LA in the FC.

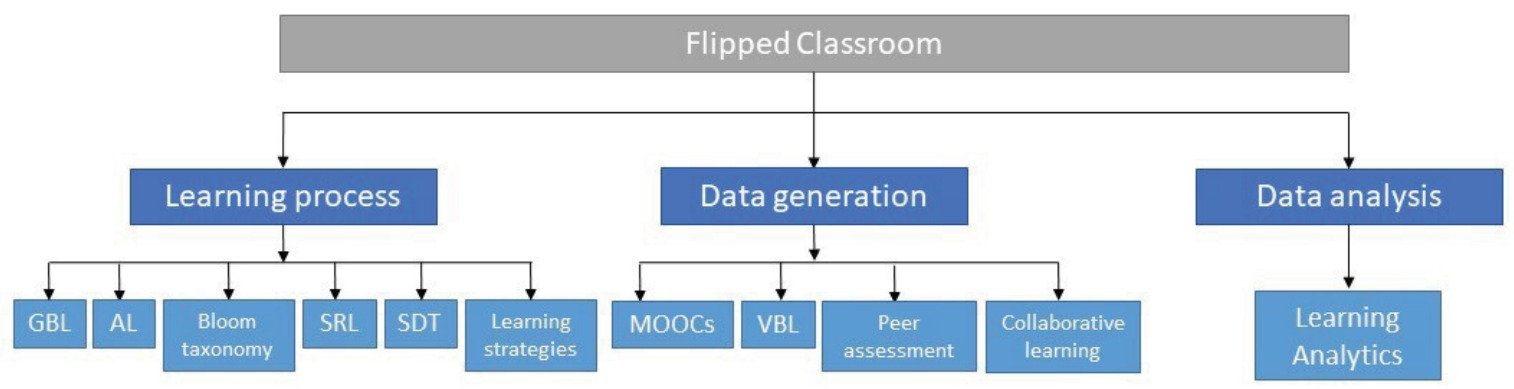

Figure 7: Theoretical framework for the structuration of FC using LA 
We have determined that we can sort out the theoretical background for integrating LA in the FC in a three-tier distribution: learning process, data generation, and data analysis. By data generation, we underline the presentation of educational tools that generate data such as MOOCs (e.g Ng and Xie, 2017), VBL (Garrick, 2018) or specific LA tools (e.g Redondo et al., 2015). The dominance of LMS traces in data collection illustrates this focus on online tools for FC management and data collection. A minority of studies also presented the theoretical background behind peer assessment (Lin, 2019) or collaborative learning (Hwang and Chen, 2019) for research that specifically focused on these issues.

Finally, a significant 28 out of 49 articles presented an expanded context that also included educational theories to map the learning experience of the student population, which we map under learning process. Self-Regulated Learning (SRL) takes the greatest share of mentions, with 10 articles represented (e.g. AlJarrah, Thomas, and Shehab, 2018; Silva et al., 2018; Saint et al., 2018; Fincham et al., 2018; Jovanović et al., 2017; Sun, Lu, and Xie, 2016; Pardo et al., 2015). The SRL theory (Pintrich and Garcia, 1994) establishes that students can have better learning outcomes, cognition, and behaviour with planning, monitoring, and regulating strategies, but also that these strategies are not inherent traits but aptitudes that can be trained. Therefore, many studies use this theory as a means to encourage the use of LA in the FC to support students' self-regulation in learning and metacognitive strategies. In that regard, potential for increased benefit for SRL in students appears a strong trend.

In the same vein, four articles reference the notion of learning strategies with the aim to identify specific learning pathways through LA (Fincham et al., 2018; Jovanović et al., 2017, Jovanović et al., 2019; Schwarzenberg, Navon and Pérez-Sanagustín, 2019). Another essential cognitive theory appears in the form of the Self-Determination Theory (SDT), which posits the existence of motivation as a continuum based on a sense of competence, autonomy, and relatedness. This theory, as developed by Deci and Ryan (1985), appears in two articles (Sergis, Sampson, and Pelliccione, 2018; Isomöttönen and Tirronen, 2017). Finally, a smattering of studies reference other educational theories to analyse the efficiency of the FC. These studies reference for example the principle of active learning (Kaw et al., 2019; Hui et al., 2018), Bloom's taxonomy (Hwang and Chen, 2019; Giannakos, Chorianopoulos, and Chrisochoides, 2015), and Game-Based Learning (GBL) (Chu, Wang and Wang, 2019).

Based on the aforementioned elements, we can now question what were the learning outcomes observed by integrating LA in the FC. Most studies, 18 out of 49, focused on the correlation between students' learning performances and online activity, and found positive results correlating the highest grades and pass rates with the most active learning strategies (e.g. Hsiao et al., 2018; Martínez-Muñoz and Pulido, 2015; Smallhorn, 2017; Mouri, Uosaki and Ogata, 2018). Two studies among these especially highlighted the positive impact of students engaging with online feedback on their learning process on higher levels of SRL and better learning outcomes (Matcha et al., 2019; Silva et al., 2018). Some studies succeeded in identifying high performing student groups by correlating their online engagement and assessment performance (Reidsema et al., 2017; Saint, Gašević and Pardo, 2018), and showed that online environments encouraged students' participation in the FC (Hsu, 2019), especially collaborative learning and discussions. Studies, which also investigated the students' perception of the process, found high level of students' satisfaction in the learning experience (Lei et al., 2017; Corrias and Hong, 2015; Smallhorn, 2017), and reduced levels of anxiety in low-performing students (Chu, Wand and Wang, 2019).

The second most represented outcome of these studies, with 16 articles, was the use of LA as a means to improve the learning experience and the FC process. Such studies offered LA to help teachers improve the course (e.g. Van Leeuwen, 2018), to enable adaptive learning by selecting the best material and exercises according to the student's profile (e.g. Xiao, Pham and Wang, 2015), or to select the best learning materials and videos (e.g. Lau et al., 2018; Kravchenko and Cass, 2017). Within this group, some studies focused on the temporality of students' engagement with the learning material to issue recommendations for adaptive learning and improvement of the learning material (e.g. Garrick, 2018; Silva et al. 2018).

Finally, several studies presented positive learning outcomes in the capacity of LA to predict students' success or risk of dropout and monitor interventions, but this approach appears more recent, represented only in the past two years. Five articles presented strategies aligning with these objectives (Hui and al., 2018; Van Leeuwen, 2018; Nouri, Uosaki and Ogata, 2019; Schwarzenberg, Navon and Pérez-Sanagustín, 2019), developing predictive models or supporting teachers' intervention towards students at risk of under-performing. 
Therefore, the majority of studies presented positive results and made a compelling argument to the idea that LA support the FC experience and result in better success in the FC, and better monitoring and support of online engagement. Furthermore, these studies showed that using LA in the FC could reinforce the method by providing an adaptive learning framework to students, and encourage SRL. Finally, the potential for the use of predictive statistics was observed, and their capacity to predict success and identify vulnerable students.

\subsection{What were the main limitations in the use of LA in the FC?}

Although the impact of implementing the FC and the use of LA seemed overwhelmingly positive, this review allowed us to observe several limitations, especially issues connected to students' engagement with the learning material, limited use of feedback and LA on learning strategies, and students' difficulties to adopt the methodology. Twelve articles out of 49 presented similar issues, which we can decompose into the following specific problematics:

- Quality of engagement: several studies (e.g. Marasco et al., 2018) underlined that the measures from LA were mostly click-based, and did not evaluate the quality of students' engagement with the learning material.

- A utilitarian engagement: two studies (Ayres et al., 2018; Smallhorn, 2017) underline the fact that many students only performed the learning activities before the exam, or if grades were attached to them.

- Specific difficulties: some studies underlined the difficulties of students to self-regulate their learning process (Isomöttönen and Tirronen, 2017), or the reluctance of already high-performing students to adopt new learning methodologies (Pardo et al., 2015).

Some studies also underlined negative or non-significant results. For example, one pointed no statistical difference between passing and failing students (Gelan et al., 2018), while another one no correlation between success rate and time spent online (Yang, Wu and Cao, 2016). One had a disappointing pass rate due to lack of quality engagement with the learning materials (Lei et al., 2017), or no effect for the highest performing students (Jovanovic et al., 2017). One study could not find correlation between the use of VBL for capturing lectures and the evaluated results in their students, and had to conclude that their use of VBL did not yield any improvement for them (Williams, Aguilar-Roca and O'Dowd, 2016).

Finally, we observed the fact that only 10 articles presented studies where direct feedback or visualizations were part of the experimental design. Of these studies, one limited access to the analytics information to teachers so that they could intervene (Fernández, Merino, and Kloos, 2019), but the rest provided students direct access to feedback information, or their own learning analytics. Most of these studies allowed students access to their analytics in real time with a review of their engagement and completion successes (Giannakos, Chorianopoulos and Chrisochoides, 2015; Redondo et al., 2015; Hsu, 2019; Kaw et al., 2019; Jovanović et al., 2017). One study provided a learning bulletin at the end of the session (Silva et al., 2018). Two studies used both methods conjoined, giving students both a real-time analytics feedback and a personalized dashboard information, and personalized feedback at the end of the session (Jovanovic et al., 2019; Matcha et al., 2019). These examples show that direct feedback is also a major element in the development of LA, and that there is room to improve its use in the FC and exploit its potential to develop fully adaptive learning.

\section{Discussion}

\subsection{Educational data mining, LA, and educational models}

This scoping review shows that there is great potential in using LA in the FC, especially as a means to reinforce the efficiency of the methodology. Improvements in technology allow instant access to a wide range of educational data. However, we observed that, because of its reliance on online educational data, most studies focused on the pre-class preparation, and approached the post-class process only through students' summative assessments and results. There is, therefore, a clear lack of research investigating the in-class process and activities.

Furthermore, to inform and improve educational practice, key researchers have underlined the need for LA to be rooted in research on learning and teaching (Gelan et al., 2018), and our research showed that studies in applying LA to the FC focused so far mostly on the technical aspects and did not articulate their results with indepth learning theories. In that regard, the most promising angle we observed is the use of SRL theory to analyse and understand patterns in students' behaviour and learning strategies. There is, therefore, an under- 
investigated potential in putting more emphasis on SRL, and giving better feedback and access to LA to students with the aim to develop fully adaptive learning.

Finally, if we take into account the most common types of LA, our current study allows us to discuss what the major evolutions in the application of LA in FCs could be through observation of the LA types employed in our 49 studies. Figure 8 presents the four types of LA that serve as a basis for our discussion on the potential future of LA in the FC.
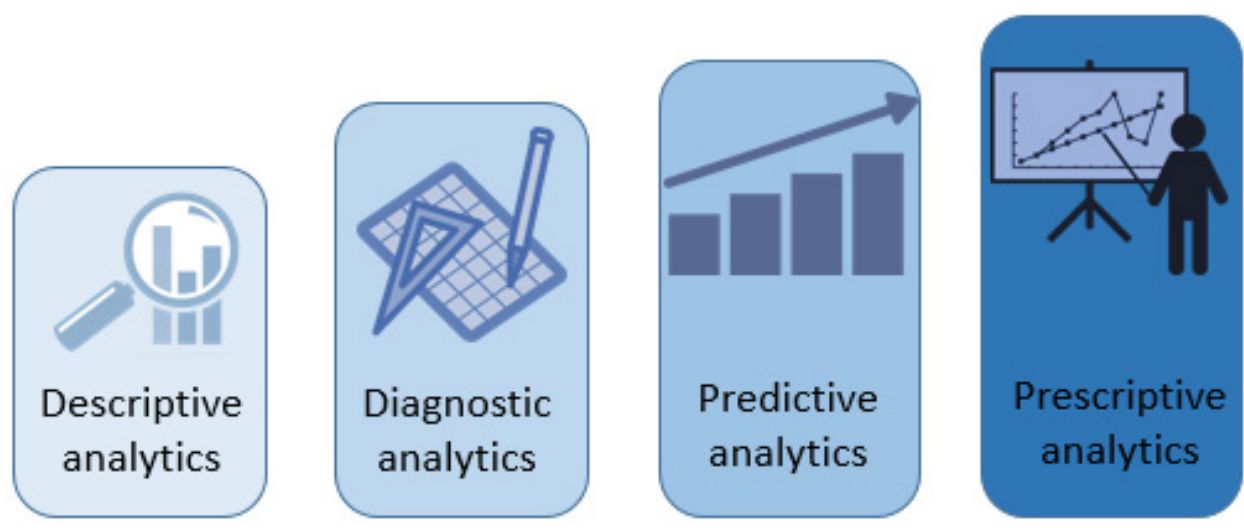

Figure 8: The four types of Learning Analytics

In the reviewed studies, we observed the following applications of LA:

- Descriptive analytics ask, "What happened?" As we observed earlier, most studies approached LA in a general descriptive manner based on educational data mining. LA in these many instances relied on the use of LMS traces to monitor students' engagement with the learning platform, and the measure of their learning performance.

- Diagnostic analytics show interest into "Why did it happen?" They intervene in observing students' patterns of learning and correlating explication variables to the observations. They are usually used in studies that correlate students' success and improved learning experiences with experience in the FC and use of LA.

- Predictive analytics are one of the huge potential in the FC and raise the issue "What will happen?" Studies with interest in predictive analysis try especially to allow early intervention to adjust the course of a curriculum or anticipate risk of dropout in students.

- Prescriptive analytics appear as the last potential expansion, and try to focus on "How can we make it happen?" In our corpus, this was observed through a combination of data mining, cluster analysis or use of neural networks in an attempt to identify specific student groups or learning strategies. This classification work should allow educators in the future to identify and support the best practices. In the future however, more potential is to be found into combining predictive analysis with targeted recommendations and visualizations.

Therefore, we will argue that our scoping review allowed us to present a solid overview of the current state of $\mathrm{LA}$ in the FC and to project that the process is bound to undergo further development. We especially anticipate interest in predictive analytics and prescription analytics, resulting in increased use of instant feedback, and user visualizations and targeted recommendations.

\subsection{Educational outcomes and limitations}

The majority of studies presented positive learning outcomes and the capacity to predict student performance based on their online engagement with the learning materials and with student-teacher or peer-activities. However, our analysis showed that there are still fields that remain underexploited regarding LA in the FC.

Few studies tried to investigate the long-term effects of the FC, and used only students' results on a given course. Indeed, some groups showed no significant improvement in short-term assessment in the FC, but improved results in long-term assessment (Hsiao et al., 2018). Similarly, few studies investigated the effect on different populations of students. Some only used the video metrics without evaluating individual users' engagement (e.g. Kravchenko and Cass, 2017), and only two studies presented a different population. Kaw et al. (2019) 
investigated students "other than white male" and Ng and Xie (2017) showed that the FC was slightly more efficient with female students. Other studies insisted on the fact that their model affected a limited number of subjects and could not yet be generalized to all students (Chu, Wand and Wang, 2019).

Finally, our research shows that there are still issues that need to be resolved to implement efficiently LA in the FC. For example, click-based data can only measure superficial online engagement and should be completed by either qualitative data from interviews or students' social interactions. Marasco et al. (2018) even suggested that the whole system of evaluation should evolve to fit the new paradigm.

\section{Conclusion}

Faced with the necessity to promote and develop active learning, educational institutions have turned massively towards the FC to encourage self-regulated learning in students. As the efficiency of the FC became well established, the potential of improving the FC by using LA seems a logical step forward. By conducting a scoping review, we found that most articles on LA for FCs were published after 2017 describing interventions mainly in STEM fields. Moreover, LMS and VBL traces were broadly used for descriptive or diagnostic analytics, which were also the most common type of analytics employed. Our literature review revealed also that there is potential in using LA in the FC, especially as a means to predict students' learning outcome and to support adaptive learning and improvement on the curriculum. However, further development and long-term studies are necessary to encourage self-directed learning in students and to develop the whole of the FC (pre-, in- and post-class sessions) for a more diverse population of students. We anticipate an increased and expanded use of LA to come, with focus on predictive analytics and prescription analytics, resulting in increased use of instant feedback, and user visualizations and targeted recommendations. We also anticipate that LA will expand beyond data mining to correlate student performance and online engagement with the aim to include a wider range of possibilities of interventions and adaptation of the learning experience.

\section{Acknowledgements}

This research was funded by the FLIP2G project. This project has been funded with the support of the Erasmus+ programme of the European Union. This paper reflects the views only of the authors, and the Commission cannot be held responsible for any use which may be made of the information contained therein.

\section{References}

\section{Study Corpus}

AlJarrah, A., Thomas, M. K., and Shehab, M., 2018. Investigating temporal access in a flipped classroom: procrastination persists. International Journal of Educational Technology in Higher Education, 15(1), 1.

Ayres, I. M. E., Fisteus, J. A., Uguina-Gadella, L., Hoyos, C. A., and Kloos, C., D. (2018. Uncovering Flipped-Classroom Problems at an Engineering Course on Systems Architecture Through Data-Driven Learning Design. The International journal of engineering education, 34(3), 865-878.

Chu, H. C., Wang, C. C. and Wang, L., 2019. Impacts of Concept Map-Based Collaborative Mobile Gaming on English Grammar Learning Performance and Behaviors. Journal of Educational Technology \& Society, 22(2), 86-100.

Corrias, A. and Hong, J. G. C., 2015. Design and implementation of a flipped classroom learning environment in the biomedical engineering context. In 2015 37th Annual International Conference of the IEEE Engineering in Medicine and Biology Society (EMBC) IEEE. pp. 3985-3988.

Fincham, O. E., Gasevic, D. V., Jovanovic, J. M., and Pardo, A., 2018. From study tactics to learning strategies: an analytical method for extracting interpretable representations. IEEE Transactions on Learning Technologies. Vol.12(1), pp.59-72

Garrick, R., 2018, Flipped Classroom Video Analytics, 2018 ASEE Annual Conference \& Exposition, Salt Lake City, Utah. American Society for Engineering Education, pp1-9.

Gelan, A., Fastré, G., Verjans, M., Martin, N., Janssenswillen, G., Creemers, M. and Thomas, M., 2018. Affordances and limitations of learning analytics for computer-assisted language learning: a case study of the VITAL project. Computer Assisted Language Learning, 31(3), 294-319.

Giannakos, M. and Chrisochoides, N., 2014. Challenges and perspectives in an undergraduate flipped classroom experience: Looking through the lens of learning analytics. In 2014 IEEE Frontiers in Education Conference (FIE) Proceedings IEEE. pp. 1-5.

Giannakos, M. N., Chorianopoulos, K. and Chrisochoides, N., 2015. Making sense of video analytics: Lessons learned from clickstream interactions, attitudes, and learning outcome in a video-assisted course. The International Review of Research in Open and Distributed Learning, 16(1).

Gilliland, K., O., 2017. The flipped classroom and learning analytics in histology. Medical Science Educator, 27(1), 9-13. 
Hecking, T., Dimitrova, V., Mitrovic, A. and Ulrich Hoppe, U. (2017). Using network-text analysis to characterise learner engagement in active video watching. In ICCE 2017 Main Conference Proceedings Asia-Pacific Society for Computers in Education. pp. 326-335.

Hsiao, C. C., Huang, J. C., Huang, A. Y., Lu, O. H., Yin, C. J. and Yang, S. J.,2018. Exploring the effects of online learning behaviors on short-term and long-term learning outcomes in flipped classrooms. Interactive Learning Environments, 1-18.

Hsu, T., 2019. The different effects of daily-life instant response social media and an educational feedback system on flipped learning: from the evidence of behavioral analysis. Interactive Learning Environments, 1-20.

Hui, Y. K., Mai, B., Qian, S. and Kwok, L. F.,2018. Cultivating better learning attitudes: a preliminary longitudinal study. Open Learning: The Journal of Open, Distance and e-Learning, 33(2), 155-170.

Hwang, G. J. and Chen, P. Y., 2019. Effects of a collective problem-solving promotion-based flipped classroom on students' learning performances and interactive patterns. Interactive Learning Environments, 1-16.

Isomöttönen, V. and Tirronen, V. (2017). Flipping and blending-an action research project on improving a functional programming course. ACM Transactions on Computing Education (TOCE), 17(1), 1.

Ji, Y., and Han, Y, 2019. Monitoring Indicators of the Flipped Classroom Learning Process based on Data Mining-Taking the Course of" Virtual Reality Technology" as an Example. International Journal of Emerging Technologies in Learning, 14(3).

Jovanović, J., Gašević, D., Dawson, S., Pardo, A. and Mirriahi, N. 2017. Learning analytics to unveil learning strategies in a flipped classroom. The Internet and Higher Education, 33(4), 74-85.

Jovanovic, J., Mirriahi, N., Gašević, D., Dawson, S. and Pardo, A, 2019. Predictive power of regularity of pre-class activities in a flipped classroom. Computers \& Education, 134, 156-168.

Kaw, A., Clark, R., Delgado, E. and Abate, N., 2019 Analyzing the use of adaptive learning in a flipped classroom for preclass learning. Computer Applications in Engineering Education.

Kravchenko, M. and Cass, A., 2017. Attention retention: Ensuring your educational content is engaging your students. In International Conference on Smart Education and Smart E-Learning (pp. 358-370). Springer, Cham.

Lau, K., Farooque, P., Leydon, G., Schwartz, M., Sadler, R. and Moeller, J., 2018. Using learning analytics to evaluate a videobased lecture series. Medical teacher, 40(1), 91-98.

Lei, C., Yau, C., Lui, K., Yum, P., Tam, V., Yuen, A. and Lam, E., 2017. Teaching Internet of Things: Enhancing learning efficiency via full-semester flipped classroom. In 2017 IEEE 6th International Conference on Teaching, Assessment, and Learning for Engineering (TALE) IEEE. pp. 56-60.

Lin, C., 2019. An online peer assessment approach to supporting mind-mapping flipped learning activities for college English writing courses. Journal of Computers in Education, 6(3), 385-415.

Lin, C. and Hwang, G., 2018. A learning analytics approach to investigating factors affecting EFL students' oral performance in a flipped classroom. Journal of Educational Technology \& Society, 21(2), 205-219.

Marasco, E. A., \& Moshirpour, M., \& Moussavi, M., \& Behjat, L. and Amannejad, Y, 2018. Evidence-based Best Practices for First-year Blended Learning Implementation, 2018 ASEE Annual Conference \& Exposition, Salt Lake City, Utah, pp110.

Martínez, J. A., Campuzano, J., Sancho-Vinuesa, T. and Valderrama, E., 2019. Predicting student performance over time. A case study for a blended-learning engineering course In: CEUR Workshop Proceedings, Vol. 2415, 2019, p. 43-55.

Martínez-Muñoz, G., and Pulido, E., 2015. Using a SPOC to flip the classroom. In 2015 IEEE Global Engineering Education Conference (EDUCON) IEEE. pp. 431-436.

Matcha, W., Gašević, D., Uzir, N., Jovanović, J. and Pardo, A., 2019. Analytics of Learning Strategies: Associations with Academic Performance and Feedback. In Proceedings of the 9th International Conference on Learning Analytics \& Knowledge ACM. pp. 461-470.

Mouri, K., Uosaki, N. and Ogata, H., 2018. Learning Analytics for Supporting Seamless Language Learning using E-book with Ubiquitous Learning System. Educational Technology \& Society, 21 (2), 150-163.

$\mathrm{Ng}$, V. and Xie, S., 2017. Student Engagement With Video-Watching and Flipped Class Behaviors. In ICEL 2017-Proceedings of the 12th International Conference on e-Learning Academic Conferences and publishing limited., pp. 163-168).

Nouri, J., Saqr, M. and Fors, U., 2019. Predicting performance of students in a flipped classroom using machine learning: towards automated data-driven formative feedback. In 10th International Conference on Education, Training and Informatics (ICETI 2019).

Pardo, A., Mirriahi, N., Dawson, S., Zhao, Y., Zhao, A. and Gašević, D., 2015. Identifying learning strategies associated with active use of video annotation software. In Proceedings of the Fifth International Conference on Learning Analytics and Knowledge ACM. pp. 255-259.

Poon, L., Kong, S., Wong, M. and Yau, 2017. Mining sequential patterns of students' access on learning management system. In International conference on data mining and big data Springer, Cham. pp. 191-198.

Redondo, D., Muñoz-Merino, P., Ruipérez-Valiente, J., Delgado Kloos, C., Pijeira Díaz, H. and Santofimia Ruiz, J., 2015. Combining Learning Analytics and the Flipped Classroom in a MOOC of maths.

Reidsema, C., Khosravi, H., Fleming, M., Kavanagh, L., Achilles, N. and Fink, E., 2017. Analysing the learning pathways of students in a large flipped engineering course.

Rubio-Fernández, A., Muñoz-Merino, P. J., \& Delgado Kloos, C. (2019). A learning analytics tool for the support of the flipped classroom. Computer Applications in Engineering Education, 27(5), 1168-1185. 
Saint, J., Gašević, D. and Pardo, A., 2018. Detecting Learning Strategies Through Process Mining. In European Conference on Technology Enhanced Learning Springer, Cham pp. 385-398.

Schwarzenberg, P., Navon, J. and Pérez-Sanagustín, M., 2019. Models to provide guidance in flipped classes using online activity. Journal of Computing in Higher Education, 1-25.

Sergis, S., Sampson, D. G. and Pelliccione, L., 2018. Investigating the impact of Flipped Classroom on students' learning experiences: A Self-Determination Theory approach. Computers in Human Behavior, 78, 368-378.

Silva, J. C. S., Zambom, E., Rodrigues, R. L., Ramos, J. L. C. and de Souza, F. D. F., 2018. Effects of Learning Analytics on Students' Self-Regulated Learning in Flipped Classroom. International Journal of Information and Communication Technology Education (IJICTE), 14(3), 91-107.

Smallhorn, M., 2017. The flipped classroom: A learning model to increase student engagement not academic achievement. Student Success, 8(2), 43-53.

Sun, Z., Lu, L. and Xie, K., 2016. The Effects of Self-Regulated Learning on Students' Performance Trajectory in the Flipped Math Classroom. Singapore: International Society of the Learning Sciences. ICLS 2016 Proceedings, pp66-73.

Van Leeuwen, A., 2018. Teachers' perceptions of the usability of learning analytics reports in a flipped university course: when and how does information become actionable knowledge?. Educational Technology Research and Development, 1-22.

Williams, A. E., Aguilar-Roca, N. M. and O'Dowd, D. K., 2016. Lecture capture podcasts: differential student use and performance in a large introductory course. Educational Technology Research and Development, 64(1), 1-12.

Xiao, X., Pham, P. and Wang, J., 2015. AttentiveLearner: adaptive mobile MOOC learning via implicit cognitive states inference. In Proceedings of the 2015 ACM on International Conference on Multimodal Interaction ACM, pp. 373374.

Yamada, Y. and Hirakawa, M., 2015. A case Study of analyzing Logs of LMS in Flipped Classroom. In 2015 IIAI 4th International Congress on Advanced Applied Informatics IEEE. pp. 374-378.

Yang, Y., Wu, H. and Cao, J., 2016. Smartlearn: Predicting learning performance and discovering smart learning strategies in flipped classroom. In 2016 International Conference on Orange Technologies (ICOT) IEEE, pp. 92-95.

\section{Other references}

Abeysekera, L. and Dawson, P., 2015. Motivation and cognitive load in the flipped classroom: Definition, rationale and a call for research. Higher Education Research \& Development, 34(1), 1-14.

Arksey, H. and O'Malley, L., 2005. Scoping studies: towards a methodological framework. International journal of social research methodology, 8(1), 19-32.

Bergmann, J. and Sams, A., 2009. Remixing chemistry class: Two Colorado teachers make vodcasts of their lectures to free up class time for hands-on activities. Learning \& Leading with Technology, 36(4), 22-27.

Bishop, J. and Verleger, M. A. (2013. The flipped classroom: A survey of the research. In 2013 ASEE national conference proceedings, Atlanta, GA, Vol. 30, No. 9, pp. 1-18.

Doko, E. and Bexheti, L. A.,2018. A systematic mapping study of educational technologies based on educational data mining and learning analytics. In 2018 7th Mediterranean Conference on Embedded Computing (MECO) (pp. 1-4). IEEE.

Fernández, A. R., Merino, P. J. M., and Kloos, C. D., 2018. Scenarios for the application of learning analytics and the flipped classroom. In 2018 IEEE Global Engineering Education Conference (EDUCON) (pp. 1619-1628). IEEE.

Frey, B., 2018. The SAGE encyclopaedia of educational research, measurement, and evaluation. Thousand Oaks, California: SAGE Publications, pp 39-40.

Klemke, R., Eradze, M. and Antonaci, A., 2018. The flipped MOOC: using gamification and learning analytics in MOOC design-a conceptual approach. Education Sciences, 8(1), 25.

Lage, M., Platt, G. and Treglia, M., 2000. Inverting the classroom: A gateway to creating an inclusive learning environment. The Journal of Economic Education, 31(1), 30-43.

Lam, P., Lau, C. and Chan, C., 2019. Flipped classroom assessment: A learning process approach. Paper presented at the Multi Conference on Computer Science and Information Systems, MCCSIS 2019 - Proceedings of the International Conference on e-Learning 2019, 123-130. Retrieved from www.scopus.com

Leitner, P., Khalil, M., and Ebner, M., 2017. Learning Analytics in Higher Education-A Literature Review. In Peña-Ayala, A., 2017. Learning Analytics: Fundaments, Applications, and Trends. Springer International Publishing. Ch. 1.

Levac, D., Colquhoun, H. and O'Brien, K., 2010. Scoping studies: advancing the methodology. Implementation science, 5(1), 69.

O'Flaherty, J., and Phillips, C., 2015. The use of flipped classrooms in higher education: A scoping review. The internet and higher education, 25, 85-95.

Pintrich, P. R. and Garcia, T., 1994. Self-regulated learning in college students: Knowledge, strategies, and motivation. Student motivation, cognition, and learning: Essays in honor of Wilbert J. McKeachie, pp 113-133.

Reidsema, Carl, et al., eds. The flipped classroom: Practice and practices in higher education. Springer, 2017.

Ryan, R. M. and Deci, E. L., 2000. Self-determination theory and the facilitation of intrinsic motivation, social development, and well-being. American psychologist, 55(1), 68.

Siemens, G. and Long, P., 2011. Penetrating the fog: Analytics in learning and education. EDUCAUSE review, 46(5), 30.

Zainuddin, Z. and Halili, S. H., 2016. Flipped classroom research and trends from different fields of study. The international review of research in open and distributed learning, 17(3). 